\title{
Estratégia Saúde da Família: contribuições das equipes de saúde no cuidado nutricional da criança
}

\author{
Family Health Strategy: contributions of health teams \\ in the nutritional care of children
}

Dixis Figueroa Pedraza (http://orcid.org/0000-0002-5394-828X) ${ }^{1}$

${ }^{1}$ Universidade Estadual da Paraíba. R. das Baraúnas 351, Bodocongó. 58109-753 Campina Grande PB Brasil dixisfigueroa@gmail.com

\begin{abstract}
The scope of this article is to evaluate the structure and work processes of Family Health Strategy teams related to the nutritional care of children. It involved an assessment survey of 136 health teams in 45 municipalities in the State of Paraíba. The data on structure (availability of anthropometric equipment, supplements and technical documents) and processes (management, intersectoriality and activities of the Brazilian Breastfeeding and Feeding Strategy) were summarized in scores and classified as incipient, intermediate or advanced. Analyses were performed according to contextual characteristics of the municipality (population size and human development) and the health team (whether from the Programa Mais Médicos or conventional teams, and whether accompanied by a nutritionist or not). Nutritional care was classified at the intermediate level, with the availability of technical documents, the use of SISVAN or the e-SUS and the implementation of the Brazilian Breastfeeding and Feeding Strategy being the most deficient items. Large municipalities and expanded health teams with nutritionists had better structure and process conditions, respectively. The fragmented implementation of nutritional care calls for improvements in structure and processes for due fitness for purpose.
\end{abstract}

Key words Primary Health Care, Research into healthcare services, Nutrition programs
Resumo O objetivo deste artigo é avaliar a estrutura e o processo de trabalho de equipes de saúde da Estratégia Saúde da Família relacionados ao cuidado nutricional da criança. Pequisa avaliativa de 136 equipes de saúde em 45 municípios paraibanos. Os dados de estrutura (disponibilidade de equipamentos antropométricos, suplementos e documentos técnicos) e processo (gestão, intersetorialidade e atividades da Estratégia Amamenta e Alimenta Brasil) foram sintetizados em escores e classificados como incipiente, intermediário ou avançado. Realizaram-se análises segundo caracteristicas contextuais do município (porte populacional e desenvolvimento humano) e da equipe de saúde (se do Programa Mais Médicos ou convencional e se ampliada ou não com nutricionista). O cuidado nutricional foi classificado no nível intermediário, sendo a disponibilidade de documentos técnicos, o uso do SISVAN ou do e-SUS e a implantação da Estratégia Amamenta e Alimenta Brasil os itens mais deficitários. Municípios de porte grande e equipes de saúde ampliadas com nutricionista apresentaram melhores condições de estrutura e processo, respectivamente. A implantação fragmentada do cuidado nutricional demanda melhorias de estrutura e processo para sua qualificação.

Palavras-chave Atenção Primária à Saúde, Pesquisa sobre serviços de saúde, Programas de $\mathrm{Nu}$ trição 


\section{Introdução}

Como signatário da Década de Ação em Nutrição (2016-2025), o Brasil declarou o compromisso internacional com a melhoria da qualidade da alimentação da sua população e com a promoção de um sistema alimentar sustentável enquadrado em sei pilares: i. sistemas alimentares sustentáveis e promotores de alimentação saudável, ii. cobertura universal de ações em nutrição nos sistemas de saúde, iii. proteção social e educação nutricional, iv. comércio e investimento com foco em progressos na área de nutrição, v. ambientes seguros e propícios para a nutrição em todas as idades, e vi. revisão, fortalecimento e promoção da governança em nutrição e prestação de contas ${ }^{1}$. Nesse sentido, o segundo pilar destaca a importância dos serviços de saúde e a incorporação de ações de nutrição nos mesmos como forma de garantir avanços no estado nutricional ${ }^{1,2}$. No Brasil, a investigação sobre intervenções innovadoras, viáveis e efetivas, bem como a avaliação das ações existentes, do cuidado nutricional de menores de cinco anos na atenção básica à saúde, ainda é incipiente. Estudos com esse foco são importantes para o planejamento e aperfeiçoamento dos cuidados de nutrição nos serviços de saúde ${ }^{3}$.

As ações de cuidado nutricional são essenciais para atender as demandas geradas pelos agravos relacionados à má alimentação $0^{4}$ O desenvolvimento das mesmas de maneira qualificada contribui com os princípios do Sistema Único de Saúde (integralidade, universalidade e resolubilidade) no contexto da Atenção Primária à Saúde ${ }^{5}$, sendo a Estratégia Saúde da Família espaço privilegiado para esses fins $s^{6}$. Nesse sentido, a alimentação e nutrição representa uma área singular na qual é indispensável a colaboração entre os profissionais de saúde de diferentes formações e a atuação do nutricionista na equipe multidisciplinar $^{5-7}$. Entretanto, o cuidado nutricional na atenção básica à saúde no Brasil apresenta fragilidades associadas à inserção incipiente do nutricionista nesse nível de assistência e delegação dessa responsabilidade a um segundo plano por profissionais de outras áreas, os quais, ainda, caracterizam-se por ter conhecimentos de nutrição deficientes ${ }^{5,8}$.

Segundo Donabedian ${ }^{9}$ a análise de aspectos de estrutura e processo de trabalho é fundamental para compreender o alcance dos cuidados de saúde. Para o autor, a estrutura refere-se àquilo que é relativamente estável no sistema e propicia a prestação dos serviços (recursos financeiros, humanos e materiais) e o processo representa o conjunto de atividades e procedimentos usados no manejo dos recursos?. A importância desse modelo sistêmico tem sido sugerida para a avaliação na área de nutrição ${ }^{10}$. O estudo apresentado surge com o objetivo de avaliar a estrutura e o processo de trabalho de equipes de saúde da Estratégia Saúde da Família, relacionados ao cuidado nutricional para crianças menores de cinco anos, comparando o desempenho segundo determinantes contextuais.

\section{Métodos}

Este é um estudo quantitativo de avaliação do cuidado nutricional para crianças menores de cinco anos prestado por equipes de saúde (unidade de estudo/observação) da Estratégia Saúde da Família, a partir das condições de estrutura das unidades de saúde e do processo de trabalho das equipes de saúde. O estudo faz parte de uma pesquisa avaliativa das ações de alimentação e nutrição na Estratégia Saúde da Família no Estado da Paraíba.

O cenário do estudo compreendeu municípios do Estado da Paraíba de três contextos diferentes: i. todos os nove municípios de porte populacional de 30.000 a 149.999 habitantes que têm incentivos de custeio para a estruturação e implementação das ações de alimentação e nutrição $0^{11}$ e de prevenção da obesidade infantil no contexto do Programa Saúde na Escola ${ }^{12}$, de cobertura total da Estratégia Saúde da Família, ii. os dois municípios com população de 150.000 habitantes ou mais beneficiários dos incentivos anteriores $^{12,13}$, de cobertura parcial da Estratégia Saúde da Família, iii. municípios com população inferior a 30.000 habitantes sem os incentivos anteriores, de cobertura total da Estratégia Saúde da Família. Neste último grupo, consideraram-se os 34 municípios com equipes de saúde da Estratégia Saúde da Família do Programa Mais Médicos e convencionais e com Núcleos de Apoio à Saúde da Família vinculados tanto a equipes do Programa Mais Médicos quanto convencionais, segundo o Cadastro Nacional de Estabelecimentos de Saúde (http://cnes2.datasus.gov.br).

Para os municípios dos contextos primeiro e terceiro, o quantitativo de equipes de saúde por município foi definido considerando-se o total de equipes daquelas com menor quantidade segundo o tipo de equipe (do Programa Mais Médicos ou convencional) e a mesma quantidade de equipes naquelas com maior número, selecionadas aleatoriamente quando necessário. Esse 
mesmo critério foi estabelecido para os municípios do segundo contexto, por distritio sanitário. Dessa forma, o estudo totalizou 136 equipes de saúde (68 do Programa Mais Médicos e a mesma quantidade do modelo convencional), sendo 46 (23 de cada tipo de equipe) do primeiro contexto, 40 (20 de cada tipo de equipe) do segundo contexto e 50 ( 25 de cada tipo de equipe) do terceiro contexto.

Foram obtidas informações sobre as equipes de saúde, relacionadas às ações de alimentação e nutrição direcionadas à criança. Utilizou-se, para esses fins, questionário padronizado com perguntas fechadas previamente testado, o qual foi respondido com a participação de todos os membros da equipe de saúde, sob a liderança do enfermeiro. O questionário continha informações sobre a estrutura das unidades de saúde e a atuação das equipes de saúde em atividades relacionadas ao cuidado nutricional das crianças.

Para análise da estrutura, contemplaram-se informações sobre a disponibilidade de equipamentos antropométricos (antropômetro, estadiômetro, balança infantil e adulto), suplementos (sulfato ferroso, vitamina A) e documentos técnicos do Ministério da Saúde da área (Política Nacional de Alimentação e Nutrição, Matriz de ações de alimentação e nutrição na Atenção Básica, Marco de Referência da Vigilância Alimentar e Nutricional na Atenção Básica, Orientações para Avaliação de Marcadores de Consumo Alimentar na Atenção Básica, Orientações para a coleta e análise de dados antropométricos em serviços de saúde, Estratégia Nacional para Promoção do Aleitamento Materno e Alimentação Complementar Saudável no Sistema Único de Saúde, Guia alimentar para a população brasileira, Guia alimentar para crianças menores de dois anos, Metodologia de trabalho em grupos para ações de alimentação e nutrição na atenção básica, Saúde da criança: aleitamento materno e alimentação complementar, Saúde da criança: crescimento e desenvolvimento, Manual de condutas do Programa Nacional de Suplementação de Vitamina A, Manual de condutas do Programa Nacional de Suplementação de Ferro, Manual operacional da Estratégia NutriSUS, Manual de orientações sobre o Bolsa Família na saúde).

Para análise da atuação da equipe de saúde em atividades relacionadas ao cuidado nutricional das crianças menores de cinco anos, contemplaram-se dados pertinentes à gestão, intersetorialidade e implantação da Estratégia Amamenta e Alimenta Brasil. Os quesitos sobre a gestão e a intersetorialidade obedeceram aos fundamentos tratados no manual "Matriz de ações de alimentação e nutrição na atenção básica de saúde"14. Os parâmetros relacionados à implantação da Estratégia Amamenta e Alimenta Brasil foram estabelecidos conforme preconizado no manual da Estratégia ${ }^{15}$.

A gestão centrou-se na capacitação na área de nutrição do profissional que desenvolve/coordena as ações de cuidado nutricional e envolvimento de no mínimo um membro da equipe no planejamento das mesmas, no diagnóstico de alimentação e nutrição da população de abrangência com a identificação de áreas vulneráveis e grupos de risco, no preenchimento do Mapa de Acompanhamento do Bolsa Família, no preenchimento do Mapa Diário de Administração de Vitamina A, na interpretação de relatórios gerados a partir do SISVAN Web ou do e-SUS e na participação em reuniões com a coordenação municipal com foco na implementação das ações de nutrição nos níveis comunitário e municipal. O profissional indicado como principal ator e/ou coordenador das ações de cuidado nutricional foi considerado com capacitação adequada quando tratava-se do nutricionista ou nos casos de médicos e enfermeiros com treinamento na área de nutrição, bem como quando reconheceu-se a alternativa de trabalho multidisciplinar incluindo o nutricionista.

A intersetorialidade foi elencada sob a perspectiva de atuação em ações educativas e da vigilância alimentar e nutricional no contexto de programas estratégicos (Programa Bolsa Família e Programa Saúde na Escola), contemplando a avaliação do estado nutricional das crianças nas creches e/ou escolas, o cuidado nutricional das crianças beneficiárias do Programa Bolsa Família, a participação em orientações de alimentação e nutrição nas creches e/ou escolas e a promoção da alimentação saudável a beneficiários do Programa Bolsa Família.

A implantação da Estratégia Amamenta e Alimenta Brasil baseou-se na formação e atuação de tutores, na realização de oficinas de trabalho, na elaboração de plano de ação e na certificação da unidade de saúde.

A caracterização das equipes de saúde em relação às condições de estrutura e do processo de trabalho (dimensões) foi sintetizada por meio de um sistema de escores, pontuando-se com o valor 1 os quesitos de interesse avaliados como atendidos e zero nos outros casos. A estrutura foi formada por duas subdimensões: i. disponibilidade de equipamentos e suplementos, ii. disponibilidade de documentos técnicos do Minis- 
tério da Saúde. O processo constituiu-se de três subdimensões: i. gestão, ii. intersetorialidade, iii. implantação da Estratégia Amamenta e Alimenta Brasil. O escore de cada subdimensão derivou da soma das pontuações dos seus componentes. De forma similar, os escores da estrutura e do processo derivaram das pontuações das suas subdimensões e o escore total foi constituído da soma dos escore da estrutura e do processo. Todos os escores foram categorizados em três níveis segundo seu valor em relação ao máximo de pontos possíveis: "incipiente" $(0,0$ a 33,3\%), "intermediário" (33,4 a 66,7\%) e "avançado" (66,8 a $100,0 \%)$. O cenário "incipiente" foi entendido como proposta de "atenção simplificada", o cenário intermediário como "atenção fragmentada" e o cenário avançado como "atenção abrangente".

Os escores médios foram comparados segundo as seguintes variáveis de contexto: porte populacional do município (grande, médio/pequeno), índice de desenvolvimento humano do município (alto, médio/baixo), tipo de equipe de saúde (Programa Mais Médicos, convencional), inserção do nutricionista na equipe de saúde (equipe ampliada, equipe mínima). Municípios com 150.000 habitantes ou mais e com índice de desenvolvimento humano igual ou superior a 0,700 foram considerados de grande porte e de alto desenvolvimento humano, respectivamente. A análise dos dados foi baseada em medidas de síntese (média \pm desvio padrão), conforme a configuração da distribuição dos dados. Diferenças nas médias dos escores foram verificadas por meio do teste $t$ de Student. Estimou-se o nível de significância segundo o teste de heterogeneidade de Wald, com fins de controle do confundimento considerando as variáveis de contexto. O nível de rejeição para a hipótese de nulidade foi de $\mathrm{p}<0,05$. Todas as análises estatísticas foram conduzidas no programa estatístico Stata, versão 12.0.

A pesquisa foi aprovada pelo Comitê de Ética em Pesquisa da Universidade Estadual da Paraíba. Todos os participantes da pesquisa assinaram o Termo de Consentimento Livre e Esclarecido, como condição necessária à participação no estudo.

\section{Resultados}

Os 45 municípios selecionados representaram todas as gerências regionais de saúde do Estado da Paraíba, sendo dois de porte grande e três de índice de desenvolvimento humano alto. Das 68 equipes de saúde do Programa Mais Médicos investigadas, seis atuavam de forma ampliada com a inserção de nutricionista, com comportamento similar nas 68 equipes convencionais.

Das 136 equipes de saúde que fizeram parte do estudo, os equipamentos antropométricos estavam disponíveis em mais de $90 \%$ delas, o suplemento de vitamina A em $83,8 \%$ e o suplemento de ferro em 72,8\%. Em relação à disponibilidades de documentos técnicos observou-se que apenas três apresentaram proporção superior a $66,7 \%$ : a Política Nacional de Alimentação e Nutrição, o guia alimentar para a população brasileira e o caderno sobre aleitamento materno e alimentação complementar (Figura 1).

Além do desenvolvimento/coordenação das ações por profissional capacitado $(80,1 \%)$, o planejamento das ações $(72,1 \%)$, o preenchimento do Mapa de Acompanhamento do Bolsa Família (95,6\%), o preenchimento do Mapa Diário de Administração de Vitamina A (85,3\%), a avaliação do estado nutricional das crianças nas creches e/ou escolas (85,3\%), o cuidado nutricional das criança beneficiárias do Programa Bolsa Família $(71,3 \%)$ e as orientações de alimentação e nutrição nas creches e/ou escolas $(83,1 \%)$ foram as práticas relatadas em mais de $1 / 3$ das equipes de saúde como parte dos seus processos de trabalho (Figura 2).

Na Tabela 1 encontram-se os resultados dos escores relacionados à estrutura das unidades de saúde e ao processo de trabalho das equipes de saúde segundo o porte populacional e índice de desenvolvimento humano do município. Observa-se que a disponibilidade de documentos técnicos do Ministério da Saúde, a implantação da Estratégia Amamenta e Alimenta Brasil e ambas as dimensões, estrutura e processo de trabalho, apresentaram escores classificados como "intermediário" tanto nos municípios de grande porte quanto nos de porte pequeno/médio, bem como nos municípios de desenvolvimento humano alto e baixo/médio. A classificação do escore total foi "intermediário" em todos os contextos de interesse. Em relação às diferenças nos escores, observou-se que a disponibilidade de documentos técnicos do Ministério da Saúde e a dimensão de estrutura tiveram melhor situação nos municípios de porte grande. O escore total não apresentou diferenças entre os municípios segundo o porte populacional e o índice de desenvolvimento humano, sendo classificado como intermediário em todos os casos. 

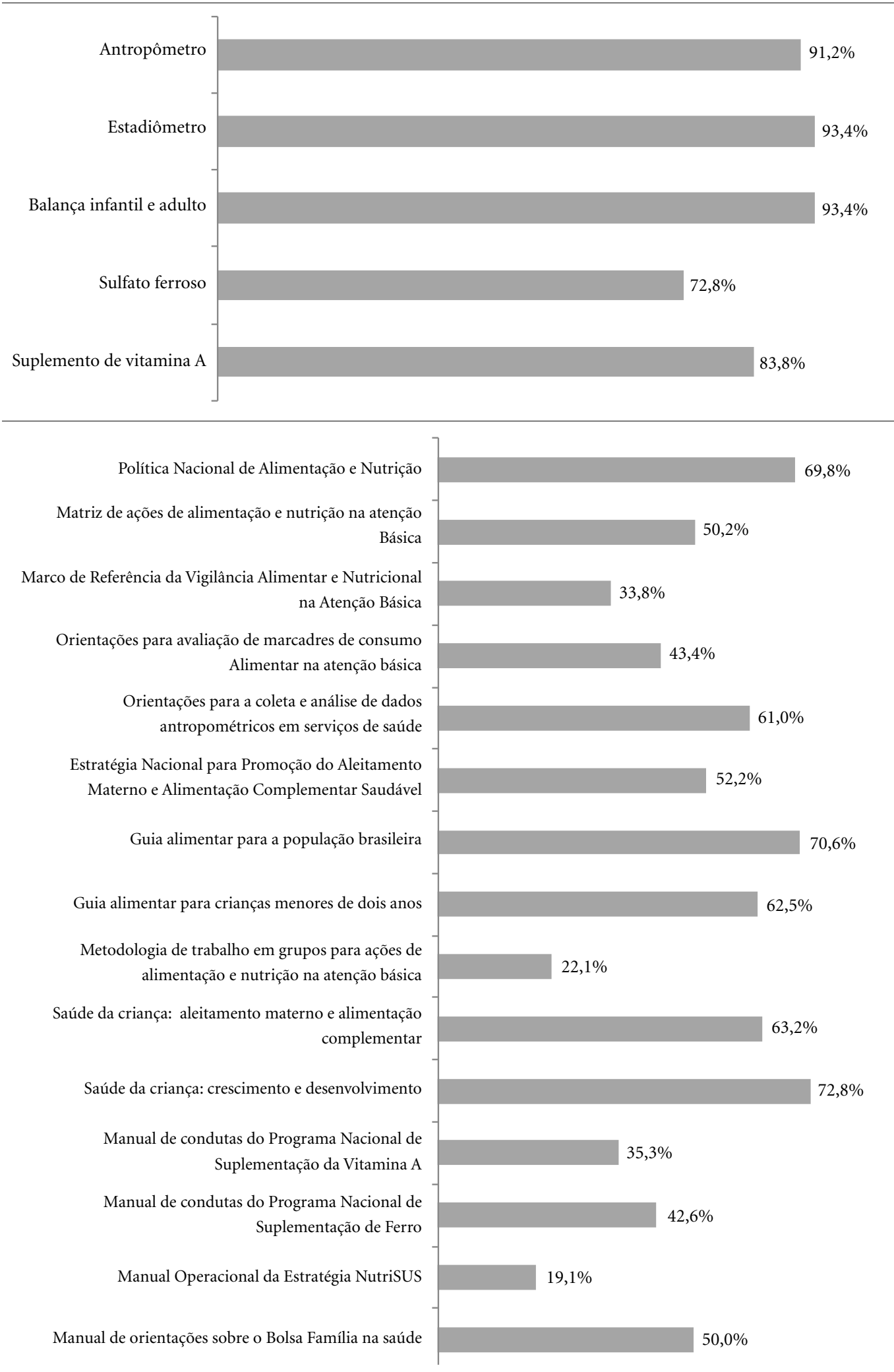

Figura 1. Proporção de unidades de saúde com disponibilidade de equipamentos antropométricos, suplementos e documentos técnicos do Ministério da Saúde, relacionados à estrutura para o cuidado nutricional das crianças menores de cinco anos de idade na Estratégia Saúde da Família no estado da Paraíba, 2017-2019.

Fonte: Elaboração própria. 


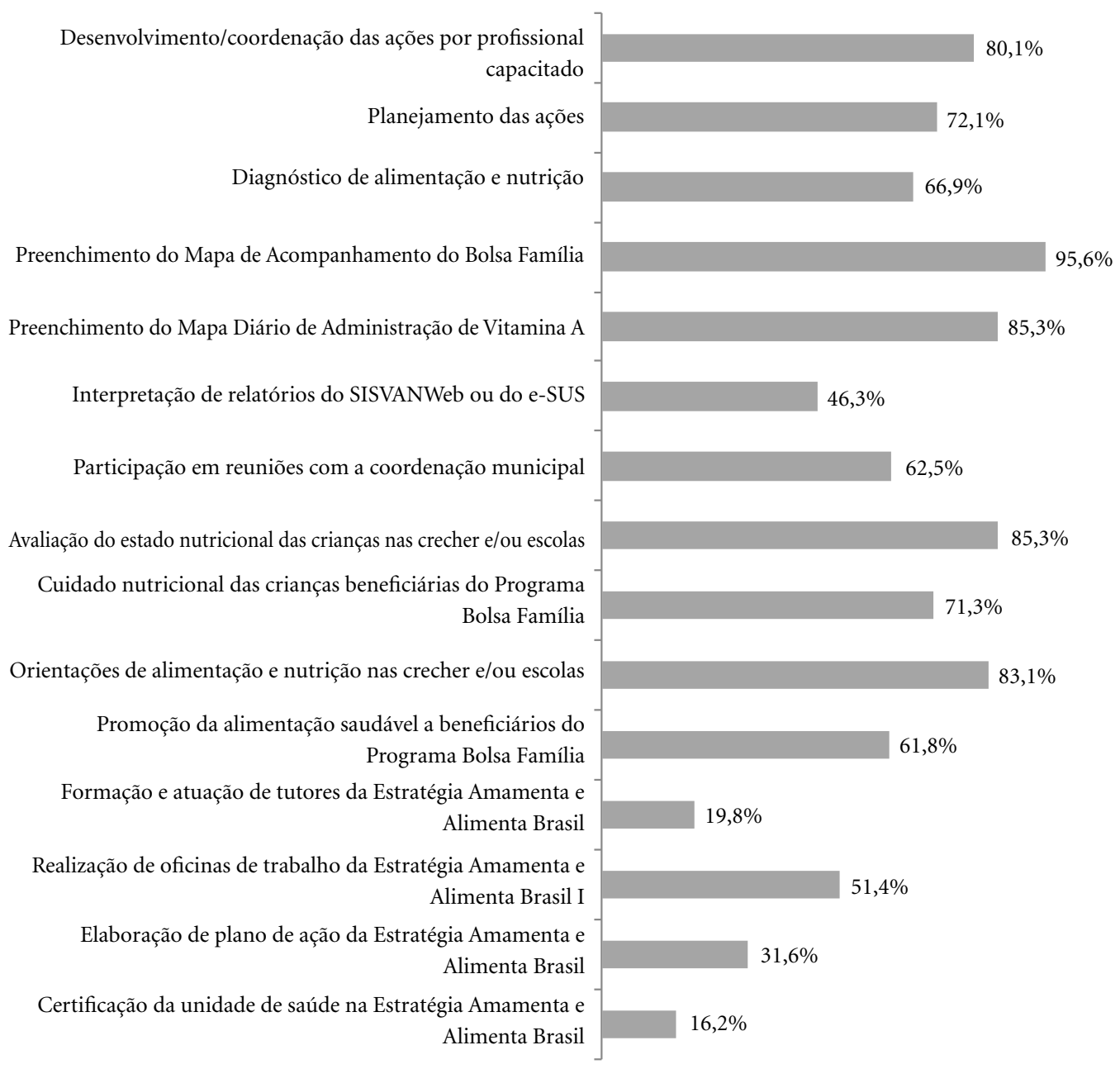

Figura 2. Proporção de equipes de saúde que desenvolvem ações de gestão, intersetorias e da Estratégia Amamenta e Alimenta Brasil, relacionadas ao processo de trabalho para o cuidado nutricional das crianças menores de cinco anos de idade na Estratégia Saúde da Família no estado da Paraíba, 2017-2019.

Fonte: Elaboração própria.

Os escores para a estrutura e o processo de trabalho de acordo com as características das equipes de saúde estão disponíveis na Tabela 2 . A disponibilidade de documentos técnicos do Ministério da Saúde e a dimensão de estrutura foram classificadas como "intermediário" para todas as situações de caracterização das equipes de saúde utilizadas. Resultado similar ao anterior foi constatado para a implantação da Estratégia Amamenta e Alimenta Brasil e para o processo de trabalho, tanto nas equipes de saúde do Programa Mais Médicos quanto nas convencionais e naquelas sem vínculo com nutricionista. A classificação do escore total foi "intermediário" em todas as categorias de contexto das equipes de saúde. $\mathrm{O}$ escore do processo de trabalho das equipes de saúde ampliadas com inserção do nutricionista foi significativamente superior ao das equipes sem a atuação desse profissional $(p=0,020)$. Não houve diferenças no escore total ao comparar as equipes de saúde atendendo a seu formato (se do Programa Mais Médicos ou convencional) e à inserção ou não do nutricionista, sendo classificado como intermediário em todos os casos. 
Tabela 1. Escores médios de indicadores da estrutura das unidades de saúde e do processo de trabalho das equipes de saúde, segundo o porte populacional e índice de desenvolvimento humano (IDH) do município, relacionados ao cuidado nutricional das crianças menores de cinco anos de idade na Estratégia Saúde da Família no Estado da Paraíba, 2017-2019.

\begin{tabular}{|c|c|c|c|c|c|c|}
\hline \multirow[t]{2}{*}{$\begin{array}{c}\text { Dimensão/Subdimensão/Variável } \\
\text { (pontuação máxima das subdimensões e } \\
\text { dimensões) }\end{array}$} & \multicolumn{2}{|c|}{$\begin{array}{c}\text { Escores médios } \\
\text { (média } \pm \text { desvio padrão) } \\
\text { segundo o porte } \\
\text { populacional do município } \\
\text { e classificação* }\end{array}$} & \multirow[t]{2}{*}{$\mathbf{p}$} & \multicolumn{2}{|c|}{$\begin{array}{c}\text { Escores médios } \\
\text { (média } \pm \text { desvio padrão) } \\
\text { segundo o IDH do } \\
\text { município e classificaçãa }{ }^{\star}\end{array}$} & \multirow[t]{2}{*}{$\mathbf{p}$} \\
\hline & Grande & $\begin{array}{l}\text { Pequeno/ } \\
\text { médio }\end{array}$ & & Alto & Baixo/médio & \\
\hline \multicolumn{7}{|c|}{ Estrutura } \\
\hline Antropômetro & $0,97 \pm 0,15^{\mathrm{I}}$ & $0,88 \pm 0,32^{\mathrm{I}}$ & 0,050 & $0,96 \pm 0,19^{I}$ & $0,88 \pm 0,32^{\mathrm{I}}$ & 0,054 \\
\hline Estadiômetro & $0,80 \pm 0,40^{\mathrm{I}}$ & $0,98 \pm 0,10^{\mathrm{I}}$ & 0,000 & $0,84 \pm 0,36^{\mathrm{I}}$ & $0,98 \pm 0,10^{\mathrm{I}}$ & 0,001 \\
\hline Balança infantil e adulto & $1,00 \pm 0,00^{\mathrm{I}}$ & $0,90 \pm 0,29^{\mathrm{I}}$ & 0,022 & $0,98 \pm 0,13^{\mathrm{I}}$ & $0,90 \pm 0,29^{I}$ & 0,042 \\
\hline Sulfato ferroso & $0,77 \pm 0,42^{\mathrm{I}}$ & $0,70 \pm 0,45^{\mathrm{I}}$ & 0,210 & $0,78 \pm 0,41^{\mathrm{I}}$ & $0,69 \pm 0,46^{\mathrm{I}}$ & 0,107 \\
\hline Suplemento de & $0,80 \pm 0,40^{\mathrm{I}}$ & $0,85 \pm 0,35^{\mathrm{I}}$ & 0,221 & $0,84 \pm 0,36^{\mathrm{I}}$ & $0,83 \pm 0,37^{\mathrm{I}}$ & 0,422 \\
\hline $\begin{array}{l}\text { Disponibilidade de equipamentos } \\
\text { antropométricos e de suplementos (5) }\end{array}$ & $4,35 \pm 0,94^{\mathrm{I}}$ & $4,34 \pm 0,88^{\mathrm{I}}$ & 0,485 & $4,42 \pm 0,87^{\mathrm{I}}$ & $4,30 \pm 0,91^{\mathrm{I}}$ & 0,215 \\
\hline Política Nacional de Alimentação e Nutrição & $0,80 \pm 0,40^{\mathrm{I}}$ & $0,65 \pm 0,47^{\mathrm{II}}$ & 0,048 & $0,78 \pm 0,41^{\mathrm{I}}$ & $0,64 \pm 0,48^{I I}$ & 0,036 \\
\hline $\begin{array}{l}\text { Matriz de ações de alimentação e nutrição } \\
\text { na Atenção Básica }\end{array}$ & $0,45 \pm 0,50^{\mathrm{II}}$ & $0,52 \pm 0,50^{\mathrm{II}}$ & 0,221 & $0,46 \pm 0,50^{\mathrm{II}}$ & $0,52 \pm 0,50^{\mathrm{II}}$ & 0,242 \\
\hline $\begin{array}{l}\text { Marco de Referência da Vigilância Alimentar } \\
\text { e Nutricional na Atenção Básica }\end{array}$ & $0,45 \pm 0,42^{\mathrm{II}}$ & $0,29 \pm 0,45^{\mathrm{III}}$ & 0,038 & $0,38 \pm 0,49^{\text {II }}$ & $0,30 \pm 0,45^{\mathrm{III}}$ & 0,186 \\
\hline $\begin{array}{l}\text { Orientações para Avaliação } \\
\text { de Consumo Alimentar na }\end{array}$ & $0,57 \pm 0,50^{\mathrm{II}}$ & $0,37 \pm 0,48^{\text {II }}$ & 0,016 & $0,48 \pm 0,50^{\mathrm{II}}$ & $0,40 \pm 0,49^{\mathrm{II}}$ & 0,19 \\
\hline $\begin{array}{l}\text { Orientações para a coleta e } \\
\text { antropométricos em serviç }\end{array}$ & $0,57 \pm 0,50^{I I}$ & $0,62 \pm 0,48^{\mathrm{II}}$ & 0,294 & $0,59 \pm 0,49^{\text {II }}$ & $0,61 \pm 0,48^{\text {II }}$ & 0,396 \\
\hline $\begin{array}{l}\text { Estratégia Nacional para Promoção do } \\
\text { Aleitamento Materno e Alimentação } \\
\text { Complementar Saudável }\end{array}$ & $0,62 \pm 0,49^{I I}$ & $0,47 \pm 0,50^{\mathrm{II}}$ &, 061 & $0,55 \pm 0,50^{\text {II }}$ & $0,50 \pm 0,50^{\mathrm{II}}$ & 0,258 \\
\hline a população brasileira & $0,65 \pm 0,48^{\mathrm{II}}$ & $0,72 \pm 0,49^{\mathrm{I}}$ & 0,179 & $0,69 \pm 0,46^{\mathrm{I}}$ & $0,71 \pm 0,45^{\mathrm{I}}$ & 0,393 \\
\hline para crianças menores de & $0,65 \pm 0,48^{\mathrm{II}}$ & $0,61 \pm 0,48^{\mathrm{II}}$ & 0,350 & $0,67 \pm 0,47^{\mathrm{I}}$ & $0,59 \pm 0,49^{\text {II }}$ & 0,183 \\
\hline $\begin{array}{l}\text { Metodologia de trabalho em grupos para } \\
\text { ações de alimentação e nutrição na atenção } \\
\text { básica }\end{array}$ & $0,32 \pm 0,47^{\mathrm{III}}$ & $0,17 \pm 0,38^{\mathrm{III}}$ & 0,029 & $0,26 \pm 0,44^{\mathrm{III}}$ & $0,19 \pm 0,39^{\mathrm{III}}$ & 0,142 \\
\hline $\begin{array}{l}\text { Saúde da criança: aleitamento materno e } \\
\text { alimentação complementar }\end{array}$ & $0,70 \pm 0,46^{\mathrm{I}}$ & $0,60 \pm 0,49^{\mathrm{II}}$ & 0,147 & $0,67 \pm 0,47^{1}$ & $0,60 \pm 0,49^{I I}$ & 0,221 \\
\hline $\begin{array}{l}\text { Saúde da criança: crescimento e } \\
\text { desenvolvimento }\end{array}$ & $0,80 \pm 0,40^{\mathrm{I}}$ & $0,69 \pm 0,46^{\mathrm{I}}$ & 0,113 & $0,75 \pm 0,43^{\mathrm{I}}$ & $0,71 \pm 0,45^{\mathrm{I}}$ & 0,326 \\
\hline $\begin{array}{l}\text { Manual de condutas do Programa Nacional } \\
\text { de Suplementação de Vitamina A }\end{array}$ & $0,52 \pm 0,50^{\mathrm{II}}$ & $0,28 \pm 0,45^{\mathrm{III}}$ & 0,003 & $0,42 \pm 0,49^{\mathrm{II}}$ & $0,30 \pm 0,46^{\mathrm{III}}$ & 0,090 \\
\hline $\begin{array}{l}\text { Manual de condutas do Programa Nacional } \\
\text { de Suplementação de Ferro }\end{array}$ & $0,55 \pm 0,50^{\mathrm{II}}$ & $0,37 \pm 0,48^{\mathrm{II}}$ & 0,030 & $0,46 \pm 0,50^{\mathrm{II}}$ & $0,40 \pm 0,49^{I I}$ & 0,259 \\
\hline Manual operacional da Estratégia NutriSUS & $0,40 \pm 0,49^{\mathrm{II}}$ & $0,10 \pm 0,30^{\mathrm{III}}$ & 0,000 & $0,30 \pm 0,46^{\mathrm{III}}$ & $0,11 \pm 0,32^{\mathrm{III}}$ & 0,003 \\
\hline $\begin{array}{l}\text { Manual de orientações sobre o Bolsa Família } \\
\text { na saúde }\end{array}$ & $0,72 \pm 0,45^{\mathrm{I}}$ & $0,40 \pm 0,49^{I I}$ & 0,000 & $0,67 \pm 0,47^{1}$ & $0,39 \pm 0,49^{I I}$ & 0,001 \\
\hline $\begin{array}{l}\text { Disponibilidade de documentos técnicos } \\
\text { do Ministério da Saúde (15) }\end{array}$ & $8,80 \pm 5,04^{\mathrm{II}}$ & $6,90 \pm 4,45^{\mathrm{II}}$ & 0,017 & $8,20 \pm 4,80^{\mathrm{II}}$ & $7,00 \pm 4,50^{\mathrm{II}}$ & 0,084 \\
\hline Estrutura (20) & $13,15 \pm 5,30^{\mathrm{II}}$ & $11,28 \pm 4,66^{\mathrm{II}}$ & 0,021 & $12,61 \pm 5,01^{\mathrm{II}}$ & $11,34 \pm 4,82^{\mathrm{II}}$ & 0,072 \\
\hline
\end{tabular}


Tabela 1. Escores médios de indicadores da estrutura das unidades de saúde e do processo de trabalho das equipes de saúde, segundo o porte populacional e índice de desenvolvimento humano (IDH) do município, relacionados ao cuidado nutricional das crianças menores de cinco anos de idade na Estratégia Saúde da Família no Estado da Paraíba, 2017-2019.

\begin{tabular}{|c|c|c|c|c|c|c|}
\hline \multirow[t]{2}{*}{$\begin{array}{c}\text { Dimensão/Subdimensão/Variável } \\
\text { (pontuação máxima das subdimensões e } \\
\text { dimensões) }\end{array}$} & \multicolumn{2}{|c|}{$\begin{array}{c}\text { Escores médios } \\
\text { (média } \pm \text { desvio padrão) } \\
\text { segundo o porte } \\
\text { populacional do município } \\
\text { e classificação }{ }^{\star} \\
\end{array}$} & \multirow[t]{2}{*}{$\mathbf{p}$} & \multicolumn{2}{|c|}{$\begin{array}{c}\text { Escores médios } \\
\text { (média } \pm \text { desvio padrão) } \\
\text { segundo o IDH do } \\
\text { município e classificação }{ }^{*}\end{array}$} & \multirow[t]{2}{*}{$\mathbf{p}$} \\
\hline & Grande & $\begin{array}{l}\text { Pequeno/ } \\
\text { médio }\end{array}$ & & Alto & Baixo/médio & \\
\hline \multicolumn{7}{|c|}{ Processo de Trabalho } \\
\hline $\begin{array}{l}\text { Desenvolvimento/coordenação das ações } \\
\text { por profissional capacitado }\end{array}$ & $0,89 \pm 0,28^{\mathrm{I}}$ & $0,88 \pm 0,22^{\mathrm{I}}$ & 0,482 & $0,91 \pm 0,25^{\mathrm{I}}$ & $0,87 \pm 0,23^{\mathrm{I}}$ & 0,15 \\
\hline $\begin{array}{l}\text { Planejamento das ações de alimentação e } \\
\text { nutrição }\end{array}$ & $0,72 \pm 0,45^{\mathrm{I}}$ & $0,71 \pm 0,45^{\mathrm{I}}$ & 0,471 & $0,75 \pm 0,43^{\mathrm{I}}$ & $0,70 \pm 0,45^{\mathrm{I}}$ & 0,275 \\
\hline $\begin{array}{l}\text { Diagnóstico de alimentação e nutrição } \\
\text { da população de abrangência com a } \\
\text { identificação de áreas vulneráveis e grupos } \\
\text { de risco }\end{array}$ & $0,62 \pm 0,49^{I I}$ & $0,68 \pm 0,46^{\mathrm{I}}$ & 0,242 & $0,63 \pm 0,48^{\mathrm{II}}$ & $0,69 \pm 0,49^{I}$ & 0,252 \\
\hline imento do Mapa de & $0,97 \pm 0,15^{\mathrm{I}}$ & $0,94 \pm 0,22^{1}$ & 0,243 & $0,98 \pm 0,13^{\mathrm{I}}$ & $0,94 \pm 0,23^{\mathrm{I}}$ & 0,13 \\
\hline $\begin{array}{l}\text { Preenchim } \\
\text { Administr }\end{array}$ & $0,85 \pm 0,36^{\mathrm{I}}$ & $0,86 \pm 0,35^{\mathrm{I}}$ & 0,475 & $0,88 \pm 0,32^{\mathrm{I}}$ & $0,83 \pm 0,37^{\mathrm{I}}$ & 0,20 \\
\hline $\begin{array}{l}\text { Interpretação d } \\
\text { do SISVAN We }\end{array}$ & $0,27 \pm 0,45^{\mathrm{III}}$ & $0,54 \pm 0,50^{\mathrm{II}}$ & 0,002 & $0,30 \pm 0,46^{\mathrm{III}}$ & $0,55 \pm 0,49^{\mathrm{II}}$ & 0,020 \\
\hline $\begin{array}{l}\text { Participação em reuniões com a } \\
\text { coordenação municipal }\end{array}$ & $0,57 \pm 0,50^{\amalg I}$ & $0,64 \pm 0,48^{\mathrm{II}}$ & 0,220 & $0,65 \pm 0,48^{\mathrm{II}}$ & $0,60 \pm 0,49^{I I}$ & 0,294 \\
\hline Gestão $(7,0)$ & $4,91 \pm 1,32^{\mathrm{I}}$ & $5,28 \pm 1,69^{I}$ & 0,111 & $5,12 \pm 1,35^{\mathrm{I}}$ & $5,20 \pm 1,73^{I}$ & 0,392 \\
\hline $\begin{array}{l}\text { Avaliação do estado nutricional das crianças } \\
\text { nas creches e/ou escolas }\end{array}$ & $0,85 \pm 0,36^{\mathrm{I}}$ & $0,86 \pm 0,35^{\mathrm{I}}$ & 0,475 & $0,84 \pm 0,36^{\mathrm{I}}$ & $0,85 \pm 0,35^{\mathrm{I}}$ & 0,430 \\
\hline $\begin{array}{l}\text { Cuidado nutricional das crianças } \\
\text { beneficiárias do Programa Bolsa Família }\end{array}$ & $0,70 \pm 0,46^{\mathrm{I}}$ & $0,71 \pm 0,45^{\mathrm{I}}$ & 0,412 & $0,77 \pm 0,42^{\mathrm{I}}$ & $0,67 \pm 0,46^{\mathrm{I}}$ & 0,129 \\
\hline $\begin{array}{l}\text { Orientações de alimentação e nutrição nas } \\
\text { creches e/ou escolas }\end{array}$ & $0,75 \pm 0,43^{\mathrm{I}}$ & $0,86 \pm 0,34^{\mathrm{I}}$ & 0,053 & $0,75 \pm 0,43^{\mathrm{I}}$ & $0,88 \pm 0,32^{\mathrm{I}}$ & 0,024 \\
\hline $\begin{array}{l}\text { Promoção da alimentação saudável a } \\
\text { beneficiários do Programa Bolsa Família }\end{array}$ & $0,70 \pm 0,46^{\mathrm{I}}$ & $0,58 \pm 0,49^{\mathrm{II}}$ & 0,102 & $0,73 \pm 0,44^{\mathrm{I}}$ & $0,54 \pm 0,50^{\mathrm{II}}$ & 0,016 \\
\hline Intersetorialidade $(4,0)$ & $3,00 \pm 1,13^{\mathrm{I}}$ & $3,02 \pm 1,06^{\mathrm{I}}$ & 0,459 & $3,09 \pm 1,07^{\mathrm{I}}$ & $2,96 \pm 1,09^{I}$ & 0,246 \\
\hline Formação e atuação de tutores da Estratégia & $0,30 \pm 0,46^{\mathrm{III}}$ & $0,15 \pm 0,36^{\mathrm{III}}$ & 0,028 & $0,32 \pm 0,47^{\mathrm{III}}$ & $0,11 \pm 0,32^{\mathrm{III}}$ & 0,002 \\
\hline Realização de oficinas de trabalho & $0,47 \pm 0,50^{\amalg}$ & $0,53 \pm 0,50^{\mathrm{II}}$ & 0,277 & $0,52 \pm 0,50^{\mathrm{II}}$ & $0,51 \pm 0,50^{\mathrm{II}}$ & 0,467 \\
\hline Elaboração de plano de ação & $0,40 \pm 0,49^{\text {II }}$ & $0,28 \pm 0,45^{\mathrm{III}}$ & 0,089 & $0,40 \pm 0,49^{I I}$ & $0,26 \pm 0,44^{\mathrm{III}}$ & 0,042 \\
\hline Certificação da unidade de saúde & $0,15 \pm 0,36^{\mathrm{III}}$ & $0,16 \pm 0,37^{\mathrm{III}}$ & 0,406 & $0,17 \pm 0,38^{\mathrm{III}}$ & $0,15 \pm 0,36^{\mathrm{III}}$ & 0,390 \\
\hline $\begin{array}{l}\text { Implantação da Estratégia Amamenta e } \\
\text { Alimenta Brasil }(4,0)\end{array}$ & $1,32 \pm 1,36^{\mathrm{II}}$ & $1,13 \pm 1,32^{\mathrm{II}}$ & 0,226 & $1,42 \pm 1,48^{\mathrm{II}}$ & $1,05 \pm 1,31^{\mathrm{II}}$ & 0,056 \\
\hline Processo de trabalho (15) & $9,23 \pm 2,95^{\mathrm{II}}$ & $9,44 \pm 2,84^{\mathrm{II}}$ & 0,356 & $9,64 \pm 2,84^{\mathrm{II}}$ & $9,21 \pm 2,88^{\mathrm{II}}$ & 0,199 \\
\hline Escore total $(35,0)$ & $22,38 \pm 6,58^{\text {II }}$ & $20,71 \pm 7,16^{\mathrm{II}}$ & 0,096 & $22,26 \pm 6,60^{\mathrm{II}}$ & $20,56 \pm 6,83^{\mathrm{II}}$ & 0,078 \\
\hline
\end{tabular}

${ }^{*}$ Categorias de classificação dos escores: Iavançado (66,8 a 100,0\% da pontuação máxima), Iintermediário (33,4 a 66,7\% da pontuação máxima), "Iincinipiente ( 0,0 a $33,3 \%$ da pontuação máxima).

Fonte: Elaboração própria. 
Tabela 2. Escores médios de indicadores da estrutura das unidades de saúde e do processo de trabalho das equipes de saúde, segundo o tipo de equipe de saúde (PMM, convencional) e a inserção do nutricionista na equipe de saúde (ampliada, mínima), relacionados ao cuidado nutricional das crianças menores de cinco anos de idade na Estratégia Saúde da Família no estado da Paraíba, 2017-2019.

\begin{tabular}{|c|c|c|c|c|c|c|}
\hline \multirow[t]{2}{*}{$\begin{array}{c}\text { Dimensão/Subdimensão/Variável } \\
\text { (pontuação máxima das subdimensões e } \\
\text { dimensões) }\end{array}$} & \multicolumn{2}{|c|}{$\begin{array}{c}\text { Escores médios } \\
\text { (média } \pm \text { desvio padrão) } \\
\text { segundo o tipo de equipe de } \\
\text { saúde e classificação }{ }^{\star}\end{array}$} & \multirow[t]{2}{*}{$\mathbf{p}$} & \multicolumn{2}{|c|}{$\begin{array}{c}\text { Escores médios } \\
\text { (média } \pm \text { desvio padrão) } \\
\text { segundo o a inserção do } \\
\text { nutricionista na equipe de } \\
\text { saúde e classificação }{ }^{\star}\end{array}$} & \multirow[t]{2}{*}{$\mathbf{p}$} \\
\hline & PMM & Convencional & & Ampliada & Mínima & \\
\hline \multicolumn{7}{|c|}{ Estrutura } \\
\hline Antropômetro & $0,95 \pm 0,20^{\mathrm{I}}$ & $0,87 \pm 0,34^{\mathrm{I}}$ & 0,035 & $0,92 \pm 0,29^{\mathrm{I}}$ & $0,91 \pm 0,28^{\mathrm{I}}$ & 0,475 \\
\hline Estadiômetro & $0,94 \pm 0,23^{\mathrm{I}}$ & $0,92 \pm 0,26^{\mathrm{I}}$ & 0,366 & $1,00 \pm 0,00^{\mathrm{I}}$ & $0,92 \pm 0,26^{\mathrm{I}}$ & 0,169 \\
\hline Balança infantil e adulto & $0,93 \pm 0,26^{\mathrm{I}}$ & $0,94 \pm 0,23^{I}$ & 0,367 & $0,92 \pm 0,28^{\mathrm{I}}$ & $0,93 \pm 0,24^{\mathrm{I}}$ & 0,402 \\
\hline Sulfato ferroso & $0,75 \pm 0,43^{\mathrm{I}}$ & $0,70 \pm 0,45^{\mathrm{I}}$ & 0,283 & $0,83 \pm 0,38^{\mathrm{I}}$ & $0,71 \pm 0,45^{\mathrm{I}}$ & 0,197 \\
\hline Suplemento de vitamina A & $0,86 \pm 0,34^{\mathrm{I}}$ & $0,80 \pm 0,39^{\mathrm{I}}$ & 0,178 & $1,00 \pm 0,00^{\mathrm{I}}$ & $0,82 \pm 0,38^{\mathrm{I}}$ & 0,056 \\
\hline $\begin{array}{l}\text { Disponibilidade de equipamentos } \\
\text { antropométricos e de suplementos (5) }\end{array}$ & $4,44 \pm 0,72^{\mathrm{I}}$ & $4,25 \pm 1,04^{\mathrm{I}}$ & 0,108 & $4,66 \pm 0,49^{\mathrm{I}}$ & $4,31 \pm 0,92^{\mathrm{I}}$ & 0,098 \\
\hline Política Nacional de Alimentação e Nutrição & $0,69 \pm 0,46^{\mathrm{I}}$ & $0,70 \pm 0,45^{\mathrm{I}}$ & 0,426 & $0,75 \pm 0,45^{\mathrm{I}}$ & $0,70 \pm 0,46^{\mathrm{I}}$ & 0,343 \\
\hline $\begin{array}{l}\text { Matriz de ações de alimentação e nutrição na } \\
\text { Atenção Básica }\end{array}$ & $0,51 \pm 0,50^{\mathrm{II}}$ & $0,48 \pm 0,50^{\mathrm{II}}$ & 0,367 & $0,50 \pm 0,52^{\mathrm{II}}$ & $0,50 \pm 0,50^{\mathrm{II}}$ & 1,000 \\
\hline $\begin{array}{l}\text { Marco de Referência da Vigilância Alimentar } \\
\text { e Nutricional na Atenção Básica }\end{array}$ & $0,34 \pm 0,47^{\mathrm{II}}$ & $0,34 \pm 0,47^{\mathrm{II}}$ & 1,000 & $0,16 \pm 0,38^{\mathrm{III}}$ & $0,35 \pm 0,48^{\text {II }}$ & 0,096 \\
\hline $\begin{array}{l}\text { Orientações para Avaliação de Marcadores de } \\
\text { Consumo Alimentar na Atenção Básica }\end{array}$ & $0,40 \pm 0,49^{\mathrm{II}}$ & $0,47 \pm 0,50^{\text {II }}$ & 0,195 & $0,16 \pm 0,38^{\mathrm{III}}$ & $0,40 \pm 0,49^{\text {II }}$ & 0,061 \\
\hline $\begin{array}{l}\text { Orientações para a coleta e análise de dados } \\
\text { antropométricos em serviços de saúde }\end{array}$ & $0,59 \pm 0,49^{\mathrm{II}}$ & $0,63 \pm 0,48^{\mathrm{II}}$ & 0,301 & $0,66 \pm 0,49^{\mathrm{II}}$ & $0,60 \pm 0,49^{I I}$ & 0,339 \\
\hline $\begin{array}{l}\text { Estratégia Nacional para Promoção do } \\
\text { Aleitamento Materno e Alimentação } \\
\text { Complementar Saudável }\end{array}$ & $0,50 \pm 0,50^{\mathrm{II}}$ & $0,54 \pm 0,50^{\mathrm{II}}$ & 0,305 & $0,34 \pm 0,49^{\text {II }}$ & $0,54 \pm 0,50^{\mathrm{II}}$ & 0,086 \\
\hline Guia alimentar para a população brasileira & $0,73 \pm 0,44^{\mathrm{I}}$ & $0,67 \pm 0,47^{1}$ & 0,228 & $0,83 \pm 0,38^{\mathrm{I}}$ & $0,74 \pm 0,46^{\mathrm{I}}$ & 0,166 \\
\hline $\begin{array}{l}\text { Guia alimentar para crianças menores de dois } \\
\text { anos }\end{array}$ & $0,57 \pm 0,49^{\mathrm{II}}$ & $0,67 \pm 0,47^{1}$ & 0,109 & $0,75 \pm 0,45^{\mathrm{I}}$ & $0,65 \pm 0,48^{\text {II }}$ & 0,179 \\
\hline $\begin{array}{l}\text { Metodologia de trabalho em grupos para } \\
\text { ações de alimentação e nutrição na atenção } \\
\text { básica }\end{array}$ & $0,23 \pm 0,42^{\mathrm{III}}$ & $0,20 \pm 0,40^{\mathrm{III}}$ & 0,341 & $0,08 \pm 0,28^{\mathrm{III}}$ & $0,23 \pm 0,42^{\mathrm{III}}$ & 0,116 \\
\hline $\begin{array}{l}\text { Saúde da criança: aleitamento materno e } \\
\text { alimentação complementar }\end{array}$ & $0,60 \pm 0,49^{\text {II }}$ & $0,66 \pm 0,47^{\mathrm{II}}$ & 0,240 & $0,58 \pm 0,51^{\mathrm{II}}$ & $0,63 \pm 0,48^{\text {II }}$ & 0,357 \\
\hline $\begin{array}{l}\text { Saúde da criança: crescimento e } \\
\text { desenvolvimento }\end{array}$ & $0,71 \pm 0,45^{\mathrm{II}}$ & $0,75 \pm 0,43^{\text {II }}$ & 0,283 & $0,58 \pm 0,51^{\mathrm{II}}$ & $0,74 \pm 0,44^{\mathrm{I}}$ & 0,326 \\
\hline $\begin{array}{l}\text { Manual de condutas do Programa Nacional } \\
\text { de Suplementação de Vitamina A }\end{array}$ & $0,34 \pm 0,47^{\mathrm{II}}$ & $0,36 \pm 0,48^{\mathrm{II}}$ & 0,361 & $0,08 \pm 0,28^{\mathrm{III}}$ & $0,32 \pm 0,48^{\mathrm{III}}$ & 0,065 \\
\hline $\begin{array}{l}\text { Manual de condutas do Programa Nacional } \\
\text { de Suplementação de Ferro }\end{array}$ & $0,40 \pm 0,49^{\mathrm{II}}$ & $0,45 \pm 0,50^{\mathrm{II}}$ & 0,246 & $0,17 \pm 0,38^{\mathrm{III}}$ & $0,41 \pm 0,49^{I I}$ & 0,068 \\
\hline Manual operacional da Estratégia NutriSUS & $0,22 \pm 0,41^{\mathrm{III}}$ & $0,16 \pm 0,37^{\mathrm{III}}$ & 0,193 & $0,00 \pm 0,00^{\mathrm{III}}$ & $0,15 \pm 0,40^{\mathrm{III}}$ & 0,089 \\
\hline $\begin{array}{l}\text { Manual de orientações sobre o Bolsa Família } \\
\text { na saúde }\end{array}$ & $0,50 \pm 0,61^{\mathrm{II}}$ & $0,50 \pm 0,61^{\mathrm{II}}$ & 1,000 & $0,50 \pm 0,52^{\mathrm{II}}$ & $0,50 \pm 0,50^{\mathrm{II}}$ & 1,000 \\
\hline $\begin{array}{l}\text { Disponibilidade de documentos técnicos do } \\
\text { Ministério da Saúde (15) }\end{array}$ & $7,34 \pm 4,98^{\mathrm{II}}$ & $7,63 \pm 4,42^{\mathrm{II}}$ & 0,358 & $6,16 \pm 3,63^{\mathrm{II}}$ & $7,56 \pm 4,78^{\mathrm{II}}$ & 0,175 \\
\hline Estrutura (20) & $11,78 \pm 5,25^{\mathrm{II}}$ & $11,88 \pm 4,59^{\mathrm{II}}$ & 0,451 & $10,83 \pm 3,48^{\mathrm{II}}$ & $11,87 \pm 5,03^{\mathrm{II}}$ & 0,291 \\
\hline
\end{tabular}


Tabela 2. Escores médios de indicadores da estrutura das unidades de saúde e do processo de trabalho das equipes de saúde, segundo o tipo de equipe de saúde (PMM, convencional) e a inserção do nutricionista na equipe de saúde (ampliada, mínima), relacionados ao cuidado nutricional das crianças menores de cinco anos de idade na Estratégia Saúde da Família no estado da Paraíba, 2017-2019.

\begin{tabular}{|c|c|c|c|c|c|c|}
\hline \multirow[t]{2}{*}{$\begin{array}{c}\text { Dimensão/Subdimensão/Variável } \\
\text { (pontuação máxima das subdimensões e } \\
\text { dimensões) }\end{array}$} & \multicolumn{2}{|c|}{$\begin{array}{c}\text { Escores médios } \\
\text { (média } \pm \text { desvio padrão) } \\
\text { segundo o tipo de equipe de } \\
\text { saúde e classificação }{ }^{*}\end{array}$} & \multirow[t]{2}{*}{$\mathbf{p}$} & \multicolumn{2}{|c|}{$\begin{array}{c}\text { Escores médios } \\
\text { (média } \pm \text { desvio padrão) } \\
\text { segundo o a inserção do } \\
\text { nutricionista na equipe de } \\
\text { saúde e classificação }{ }^{*}\end{array}$} & \multirow[t]{2}{*}{$\mathbf{p}$} \\
\hline & PMM & & & Ampliada & & \\
\hline \multicolumn{7}{|c|}{ Processo de Trabalho } \\
\hline $\begin{array}{l}\text { Desenvolvimento/coordenação das ações por } \\
\text { profissional capacitado }\end{array}$ & $0,89 \pm 0,22^{\mathrm{I}}$ & $0,88 \pm 0,26^{\mathrm{I}}$ & 0,430 & $1,00 \pm 0,00^{I}$ & $0,87 \pm 0,22^{\mathrm{I}}$ & 0,050 \\
\hline $\begin{array}{l}\text { Planejamento das ações de alimentação e } \\
\text { nutrição }\end{array}$ & $0,75 \pm 0,43^{I}$ & $0,69 \pm 0,46^{\mathrm{I}}$ & 0,224 & $0,83 \pm 0,38^{I}$ & $0,70 \pm 0,45^{\mathrm{I}}$ & 0,183 \\
\hline $\begin{array}{l}\text { Diagnóstico de alimentação e nutrição da } \\
\text { população de abrangência com a identificação } \\
\text { de áreas vulneráveis e grupos de risco }\end{array}$ & $0,70 \pm 0,45^{\mathrm{I}}$ & $0,63 \pm 0,48^{\text {II }}$ & 0,183 & $0,66 \pm 0,49^{\mathrm{II}}$ & $0,67 \pm 0,47^{\mathrm{I}}$ & 0,493 \\
\hline $\begin{array}{l}\text { Preenchin } \\
\text { Acompanl }\end{array}$ & $0,95 \pm 0,20^{I}$ & $0,95 \pm 0,20^{\mathrm{I}}$ & 1,000 & $1,00 \pm 0,00^{I}$ & $0,95 \pm 0,21^{\mathrm{I}}$ & 0,219 \\
\hline Preenchime & $0,89 \pm 0,30^{I}$ & $0,80 \pm 0,39^{I}$ & 0,074 & $1,00 \pm 0,00^{\mathrm{I}}$ & $0,83 \pm 0,36^{\mathrm{I}}$ & 0,067 \\
\hline s gerados a partir do & $0,51 \pm 0,50^{\mathrm{II}}$ & $0,41 \pm 0,49^{I I}$ & 0,116 & $0,41 \pm 0,51^{\mathrm{II}}$ & $0,46 \pm 0,50^{\mathrm{II}}$ & 0,368 \\
\hline $\begin{array}{l}\text { Participação em reuniões com a coordenação } \\
\text { municipal }\end{array}$ & $0,60 \pm 0,49^{I I}$ & $0,64 \pm 0,48^{I I}$ & 0,299 & $0,91 \pm 0,28^{1}$ & $0,59 \pm 0,49^{I I}$ & 0,014 \\
\hline Gestão $(7,0)$ & $5,31 \pm 1,49^{1}$ & $5,02 \pm 1,69^{\mathrm{I}}$ & 0,148 & $5,83 \pm 1,26^{\mathrm{I}}$ & $5,10 \pm 1,61^{\mathrm{I}}$ & 0,067 \\
\hline $\begin{array}{l}\text { Avaliação do estado nutricional das crianças } \\
\text { nas creches e/ou escolas }\end{array}$ & $0,89 \pm 0,30^{\mathrm{I}}$ & $0,80 \pm 0,39^{I}$ & 0,074 & $0,83 \pm 0,38^{\mathrm{I}}$ & $0,85 \pm 0,35^{\mathrm{I}}$ & 0,421 \\
\hline $\begin{array}{l}\text { Cuidado nutricional das crianças beneficiárias } \\
\text { do Programa Bolsa Família }\end{array}$ & $0,70 \pm 0,46^{\mathrm{I}}$ & $0,72 \pm 0,45^{\mathrm{I}}$ & 0,425 & $1,00 \pm 0,00^{\mathrm{I}}$ & $0,68 \pm 0,46^{\mathrm{I}}$ & 0,010 \\
\hline $\begin{array}{l}\text { Orientações de alimentação e nutrição nas } \\
\text { creches e/ou escolas }\end{array}$ & $0,82 \pm 0,38^{I}$ & $0,83 \pm 0,37^{\mathrm{I}}$ & 0,410 & $0,75 \pm 0,45^{\mathrm{I}}$ & $0,83 \pm 0,36^{\mathrm{I}}$ & 0,219 \\
\hline $\begin{array}{l}\text { Promoção da alimentação saudável a } \\
\text { beneficiários do Programa Bolsa Família }\end{array}$ & $0,63 \pm 0,48^{\mathrm{II}}$ & $0,60 \pm 0,41^{\mathrm{II}}$ & 0,363 & $0,83 \pm 0,38^{\mathrm{I}}$ & $0,59 \pm 0,49^{\text {II }}$ & 0,045 \\
\hline Intersetorialidade $(4,0)$ & $3,05 \pm 1,06^{\mathrm{I}}$ & $2,97 \pm 1,10^{\mathrm{I}}$ & 0,318 & $3,41 \pm 0,79^{\mathrm{I}}$ & $2,97 \pm 1,10^{\mathrm{I}}$ & 0,089 \\
\hline Formação e atuação de tutores da Estratégia & $0,25 \pm 0,43^{\mathrm{III}}$ & $0,15 \pm 0,35^{\mathrm{III}}$ & 0,067 & $0,41 \pm 0,51^{\mathrm{II}}$ & $0,17 \pm 0,38^{\mathrm{III}}$ & 0,024 \\
\hline Realização de oficinas de trabalho & $0,51 \pm 0,50^{\amalg I}$ & $0,51 \pm 0,50^{\mathrm{II}}$ & 1,000 & $0,66 \pm 0,49^{I I}$ & $0,50 \pm 0,50^{\mathrm{II}}$ & 0,137 \\
\hline Elaboração de plano de ação & $0,32 \pm 0,47^{\mathrm{III}}$ & $0,30 \pm 0,46^{\mathrm{III}}$ & 0,427 & $0,41 \pm 0,51^{\mathrm{II}}$ & $0,30 \pm 0,46^{\mathrm{III}}$ & 0,218 \\
\hline Certificação da unidade de saúde & $0,25 \pm 0,43^{\mathrm{III}}$ & $0,07 \pm 0,26^{\mathrm{III}}$ & 0,002 & $0,25 \pm 0,45^{\mathrm{III}}$ & $0,15 \pm 0,32^{\mathrm{III}}$ & 0,194 \\
\hline $\begin{array}{l}\text { Implantação da Estratégia Amamenta e } \\
\text { Alimenta Brasil }(4,0)\end{array}$ & $1,34 \pm 1,45^{\mathrm{II}}$ & $1,04 \pm 1,20^{\mathrm{II}}$ & 0,100 & $1,75 \pm 1,48^{\mathrm{II}}$ & $1,13 \pm 1,31^{\mathrm{II}}$ & 0,065 \\
\hline Processo de trabalho $(15)$ & $9,71 \pm 2,80^{I I}$ & $9,10 \pm 2,90^{\mathrm{II}}$ & 0,087 & $11,00 \pm 2,00^{\mathrm{I}}$ & $9,22 \pm 2,90^{\Pi I}$ & 0,020 \\
\hline Escore total $(35,0)$ & $21,49 \pm 7,12^{\mathrm{II}}$ & $20,90 \pm 6,44^{\mathrm{II}}$ & 0,314 & $21,83 \pm 4,44^{\mathrm{II}}$ & $21,14 \pm 6,97^{\text {II }}$ & 0,369 \\
\hline
\end{tabular}

PMM: Programa Mais Médicos. * Categorias de classificação dos escores: ${ }^{\mathrm{I}}$ avançado (66,8 a 100,0\% da pontuação máxima), "iintermediário (33,4 a $66,7 \%$ da pontuação máxima), "IIincipiente ( 0,0 a $33,3 \%$ da pontuação máxima).

Fonte: Elaboração própria.

Após o ajuste das variáveis, foram confirmadas as associações estatísticas descritas nas Tabelas 1 e 2 . O único resultado diferente constatado foi para o desenvolvimento/coordenação das ações por profissional capacitado, característica que ganhou significância estatística $(p=0,047)$, com maior escore para as equipes de saúde ampliadas quando comparadas com as de composição mínima. 
O presente artigo descreve as condições de estrutura e processo de trabalho para o desenvolvimento das ações de alimentação e nutrição com foco na criança, em 136 equipes de saúde de 45 municípios do estado da Paraíba. Também analisa a adequação da estrutura e do processo de trabalho de acordo com características dos municípios e das equipes de saúde. Como resultado principal aponta-se um cenário de atenção fragmentada relativo ao cuidado nutricional da criança. Encontrou-se baixas prevalências de adequação na disponibilidade dos documentos técnicos do Ministério da Saúde, no uso do SISVAN Web ou do e-SUS e na implantação da Estratégia Amamenta e Alimenta Brasil. Tanto a estrutura quanto o processo de trabalho mostraram-se fragmentados, com melhor situação nos municípios de grande porte para a estrutura e nas equipes de saúde ampliadas com nutricionista para o processo.

Análise da implantação da atenção nutricional na Atenção Primária à Saúde no Brasil como a realizada no presente estudo, considerando a relação entre pontuações observadas e máximas permissíveis, também foi relatada em outros dois trabalhos que alcançaram achados similares ${ }^{16,17}$. Um desses estudos apontou de forma similar grau de implantação intermediário para o cuidado nutricional da criança menor de cinco anos em municípios do Estado da Paraíba ${ }^{16}$. No outro, a maioria dos municípios brasileiros $(57,7 \%)$ obteve a mesma classificação ao avaliar a atenção nutricional na Atenção Primária à Saúde no país, por meio dos dados do Programa Nacional de Melhoria do Acesso e da Qualidade da Atenção Básica $^{17}$.

Essas circunstâncias devem ser motivo de preocupação, dado que podem influenciar negativamente o diagnóstico nutricional, a vigilância alimentar e nutricional e o tratamento de problemas no crescimento ${ }^{18-21}$. Ainda, o baixo grau de implantação da atenção nutricional pode agravar a situação epidemiológica da população, que enfrenta grandes desafios associados ao processo de transição nutricional e às iniquidades em saú$\mathrm{de}^{22,23}$. Dessa forma, indica-se a necessidade de fortalecimento dos cuidados de nutrição como parte da assistência na Estratégia Saúde da Família $^{8,16-19,23}$, de maneira a potencializar as contribuições desse modelo na reorganização dos serviços e na saúde da população ${ }^{24}$. Para esses fins, a implantação da assistência nutricional e da Atenção Primária à Saúde enfrenta marcantes desafios relacionados ao financiamento insuficiente e à formação não orientada pelo modelo de atenção integral ${ }^{23,24}$, de modo que, apesar de ser limitado, o Financiamento da Ações de Alimentação e Nutrição representa um importante mecanismo para o fortalecimento da área no âmbito do Sistema Único de Saúde, contribuindo na capacitação profissional e na implementação das ações previstas na Política Nacional de Alimentação e Nutriçãó ${ }^{11,13,23}$.

Entre os itens de estrutura analisados, os documentos técnicos do Ministério da Saúde foram os que apresentaram menores proporções de disponibilidade nas unidades de saúde, o que também foi constatado por outros pesquisadores ${ }^{16,21}$. A indisponibilidade desses materiais também representa um dos principais fatores que limitam a vigilância do crescimento nos serviços públicos de saúde no Brasil ${ }^{25}$. É importante que os profissionais de saúde estejam devidamente capacitados na área de nutrição para melhorar o conhecimento e utilização de tais diretrizes como forma de garantir padronização aos procedimentos, sistematização e qualidade dos cuidados ${ }^{5,16,21,25}$. A grande quantidade de documentos técnicos do Ministério de Saúde que orientam a organização das ações de alimentação e nutrição nos serviços de saúde sinaliza claramente a importância e o empenho institucional para a implantação da atenção nutricional na Atenção Primária à Saúde, sendo necessário conhecer os motivos do negligenciamento dos mesmos, conforme discutido em outras publicações ${ }^{5,16}$.

Para o processo de trabalho, observou-se que menos de $50 \%$ das equipes de saúde utilizavam o SISVAN Web ou o e-SUS na interpretação dos relatórios gerados a partir desses sistemas. Este cenário corrobora com estudos que indicaram baixas coberturas do SISVAN, tanto na avaliação do consumo alimentar quanto do estado nutricional, o que denota a inutilização do Sistema como instrumento de gestão de ações de alimentação e nutrição na Estratégia Saúde da Família ${ }^{26-28}$. Dificuldades relacionadas a acesso à internet, estabilidade no vínculo laboral, sobrecarga de trabalho, capacitação dos profissionais, diferenças salariais, rotatividade na equipe e insuficiência de nutricionistas foram apontadas como limitantes na operacionalização das atividades do SISVAN ${ }^{26}$. A insuficiência de documentos técnicos da área de nutrição constitui também um problema na estrutura das unidades de saúde no país ${ }^{25}$, alentado neste estudo em específico para as referências que orientam as ações de vigilância alimentar e nutricional e do SISVAN, que pode ser outro 
possível obstáculo na implantação do Sistema. Ainda, destaca-se a pouca valorização por gestores e profissionais dos indicadores relacionados à alimentação e à nutrição $0^{22}$.

Dessa forma, discute-se a importância das melhorias necessárias na estrutura dos serviços de saúde e na formação e capacitação dos profissionais de saúde nas temáticas de nutrição e gestão ${ }^{8,26}$. Adicionalmente, levanta-se o questionamento sobre a necessidade da inserção do nutricionista no Sistema Único de Saúde dada sua competência técnica especializada para o uso do SISVAN, desde a coleta de dados até a execução de ações e divulgação ${ }^{26}$, e sua capacidade de gerar corresponsabilização necessária para o desenvolvimento das ações de alimentação e nutrição ${ }^{8}$.

Ainda na dimensão de processo de trabalho, evidenciou-se que Estratégia Amamenta e Alimenta Brasil não está plenamente implantada no âmbito das equipes de saúde estudadas. Um estudo sobre a implantação da Rede Amamenta Brasil, a qual antecedeu a Estratégia Amamenta e Alimenta Brasil, mas que mantêm estratégias de implementação iguais, identificou resultados similares. Nesse estudo, o grau de implantação esteve relacionado a diferentes contextos organizacionais, com influência de vários fatores, nomeadamente o apoio da gestão, o uso do SISVAN, o monitoramento dos indicadores de aleitamento materno, a rotatividade dos profissionais e a preparação profissional para o manejo do aleitamento materno, inclusive a utilização de protocolos ${ }^{29}$.

Acerca da Estratégia Amamenta e Alimenta Brasil foi possível identificar um artigo que também se preocupou com a mesma, reportando achados semelhantes no que diz respeito à implantação deficiente, motivada por fatores como falta de apoio da gestão, problemas no SISVAN e capacitação inadequada dos profissionais. Além disso, foram identificadas fragilidades nas informações dos documentos oficiais da Estratégia e estrutura física precária para o desenvolvimento das ações ${ }^{30}$. Em outra pesquisa de cunho descritivo os autores ressaltaram que os profissionais desconheciam a Estratégia Amamenta e Alimenta Brasil ${ }^{31}$. Estudos com objetivos diferentes corroboram esses resultados ao apontar deficiências dos profissionais na oferta de ações de promoção da alimentação saudável na infância, com possíveis prejuízos no potencial de crescimento e desenvolvimento da criança ${ }^{6,19,21}$.

Esses achados reforçam a necessidade de fortalecimento da Estratégia Amamenta e Alimenta Brasil, pois trata-se da principal intervenção com o propósito de capacitar os profissionais da Atenção Primária à Saúde em aleitamento materno e alimentação complementar, com fins de influenciar positivamente os indicadores de amamentação e auxiliar na obtenção das metas relacionadas aos mesmos ${ }^{15}$. Reforça-se, nesse sentido, a necessidade de mais informações sobre a Estratégia e da qualificação dos profissionais de saúde no contexto da educação permanente ${ }^{29-31}$. A capacitação em aconselhamento nutricional se traduz em melhorias no conhecimento sobre alimentação infantil e nas técnicas de aconselhamento e comunicação ${ }^{18}$, com potencial de avançar na adesão da população a escolhas nutricionais saudáveis ${ }^{3}$.

A estrutura das unidades básicas de saúde para o cuidado nutricional apresentou maiores escores nos municípios de grande porte, o que respalda a maior prevalência de adequação nesse quesito observada numa pesquisa que analisou a implantação das ações de alimentação e nutrição com base na avaliação externa do Programa Nacional de Melhoria do Acesso e da Qualidade da Atenção Básica ${ }^{17}$. A identificação desses municípios permite revelar grupos homogêneos que devem ser considerados para o planejamento e a organização de serviços, dada a relevância dos processos sociais na conformação de diferenciais intraurbanos e na distribuição dos agravos à saú$\mathrm{de}^{32}$. Essa premissa pode ser exemplificada com o caso do estado de Minas Gerais onde foi estabelecido o repasse de recursos financeiros pelo estado aos municípios para investimento na melhoria da infraestrutura e qualidade da Atenção Primária à Saúde, sendo os municípios menores os primeiros a serem contemplados. Tal medida foi postulada como via de explicação para a correlação negativa entre a estrutura das unidades de saúde (infraestrutura e equipamentos) e o porte populacional municipal, segundo resultados da Avaliação para Melhoria da Qualidade da Estratégia Saúde da Famíliaa ${ }^{33}$, diferentemente do constatado no presente estudo.

A possibilidade de dependência de municípios de menor porte, daqueles maiores, na oferta de serviços, inclusive de saúde, denota plausibilidade aos achados aqui registrados ${ }^{32}$. Dessa forma, é possível recomendar a necessidade de atenção especial aos municípios de pequeno/médio porte observados nesta pesquisa, uma vez que a estrutura inadequada das unidades de saúde dos mesmos pode restringir o escopo de ações e a qualidade do cuidado nutricional da criança, limitando a capacidade de resposta aos problemas nutricionais? . 
Equipes de saúde vinculadas à atuação de nutricionista apresentaram melhor desempenho no processo de trabalho, de forma similar ao que foi observado em estudo de âmbito nacional ${ }^{17}$. Ainda, resultados de outra pesquisa mostraram-se coesos ao registrar correlação dos recursos humanos, em termos da inserção do nutricionista à equipe de saúde e da capacitação dos outros profissionais de saúde na temática, com o grau de implantação das ações de alimentação e nutrição para o público de crianças $^{16}$. Esses achados podem decorrer da valorização da presença do nutricionista na Estratégia Saúde da Família por outros profissionais de saúde, ressaltando sua importância na qualificação da equipe, mais especificamente no atendimento às complexas demandas de alimentação e nutrição, por propiciar à área ampliar os conhecimentos, estimular o uso de materiais técnicos pedagógicos como o guia alimentar, potencializar as ações e incentivar o trabalho em conjunto com o compartilhamento de saberes e compromisso de todos os membros da equipe ${ }^{34}$. Os benefícios da inserção do nutricionista nas equipes de saúde podem depender da quantidade de pessoas assistidas, dos vínculos estabelecidos com a comunidade e da interação com os outros profissionais ${ }^{16}$. Tais constatações devem ser ajuizadas com ênfase, tendo em vista que o cuidado nutricional prestado por profissionais mais capacitados pode influenciar a qualidade e satisfação com os serviços de saúde ${ }^{16,21}$.

As variáveis analisadas neste estudo foram derivadas da aplicação de um questionário res- pondido pelos profissionais de saúde, os quais podem fazer superestimações. No entanto, o planejamento e desenvolvimento da pesquisa consideraram tais aspectos como forma de minimizar possíveis vieses. Ainda, a generalização externa dos achados é incerta e deve ser considerada como outra possível limitação do estudo. A metodologia utilizada tendo a equipe de saúde como unidade de estudo/observação possibilitou a obtenção de dados de bases primárias ainda não registrados na literatura. Destarte, os achados encontrados podem direcionar o planejamento de gestores e profissionais para a organização do cuidado nutricional da criança e dos serviços de saúde em geral.

Conclui-se que o cuidado nutricional da criança expressa um cenário de atenção fragmentado no qual a disponibilidade de documentos técnicos do Ministério da Saúde, o uso dos dados do SISVAN Web ou do e-SUS e o desenvolvimento das atividades da Estratégia Amamenta e Alimenta Brasil representaram os itens mais preocupantes. Também foi possível destacar melhor estrutura das unidades de saúde em municípios de grande porte e maior adequação no processo de trabalho nas equipes de saúde com inserção do nutricionista. Os resultados encontrados, portanto, indicam falhas na implantação das ações de alimentação e nutrição que demandam uma maior valorização e qualificação da área, tanto na gestão quanto no cuidado, que induzam a efetivação das intervenções nutricionais na Estratégia Saúde da Família.

\section{Referências}

1. Brasil. Câmara Interministerial de Segurança Alimentar e Nutricional (CAISAN). Compromissos do Brasil para a Década de Ação das Nações Unidas para a nutrição (2016-2025). Brasília: CAISAN; 2015.

2. International Food Policy Research Institute (IFPRI). Global Nutrition Report: From Promise to Impact: Ending Malnutrition by 2030. Washington: IFPRI; 2016.

3. Pereira TN, Monteiro RA, Santos LMP. Alimentación y nutrición en atención primaria en Brasil. Gac Sanit 2018; 32(3):297-303.

4. Brasil. Ministério da Saúde (MS). Secretaria de Atenção à Saúde. Departamento de Atenção Básica. Política Nacional de Alimentação e Nutrição. Brasília: MS; 2013.
5. Junqueira TS, Cotta RMM. Matriz de ações de alimentação e nutrição na Atenção Básica de Saúde: referencial para a formação do nutricionista no contexto da educação por competências. Cien Saude Colet 2014; 19(5):1459-1474.

6. Pimentel VRM, Sousa MF, Hamann EM, Mendonça AVM. Alimentação e nutrição na Estratégia Saúde da Família em cinco municípios brasileiros. Cien Saude Colet 2014; 19(1):49-58.

7. Watson K, Farrell M, Arensberg MB, Dwyer J. Nutrition as a Vital Sign: Progress Since the 1990 Multidisciplinary Nutrition Screening Initiative and Opportunities for Nursing. J Nurs Care 2014; 4:224. 
8. Rigon AS, Schmidt ST, Bógus CA. Desafios da nutrição no Sistema Único de Saúde para construção da interface entre a saúde e a segurança alimentar e nutricional. Cad Saude Publica 2016; 32(3):e00164514.

9. Donabedian A. The quality of care: how can it be assessed? JAMA 1988; 260:1743-1748.

10. Castaño LSA, Isaza EJP, Rueda JDG, Jaramillo AR, Ochoa CP. Métodos de evaluación de programas nutricionales de gran escala en América Latina: una revisión integrativa. Nutr Hosp 2014; 30(6):143-154.

11. Brasil. Ministério da Saúde (MS). Portaria no 55, de 6 de janeiro de 2017. Estabelece incentivo de custeio para a estruturação e implementação de ações de alimentação e nutrição pelas Secretarias de Saúde dos municípios que possuem população entre $30.000 \mathrm{e}$ 149.999 habitantes (IBGE) e do Distrito Federal, com base na Política Nacional de Alimentação e Nutrição (PNAN). Diário Oficial da União 2017; 18 jan.

12. Brasil. Ministério da Saúde (MS). Portaria no 2.706, de 18 de outubro de 2017. Lista os Municípios que finalizaram a adesão ao Programa Saúde na Escola para o ciclo 2017/2018 e os habilita ao recebimento do teto de recursos financeiros pactuados em Termo de Compromisso e repassa recursos financeiros para Municípios prioritários para ações de prevenção da obesidade infantil com escolares. Diário Oficial da União 2017; 20 out.

13. Brasil. Ministério da Saúde (MS). Portaria no 1.060, de 24 de maio de 2016. Altera o anexo I e II da Portaria no 1.738/GM/MS, de 19 de agosto de 2013. Diário Oficial da União 2016; 27 mai.

14. Brasil. Ministério da Saúde (MS). Matriz de ações de alimentação e nutrição na atenção básica de saúde. Brasília: MS; 2009. (Série A. Normas e Manuais Técnicos).

15. Brasil. Ministério da Saúde (MS). Estratégia Nacional para Promoção do Aleitamento Materno e Alimentação Complementar Saudável no Sistema Único de Saúde: manual de implementação. Brasília: MS; 2015.

16. Figueroa-Pedraza D. Implementation of food and nutrition actions in the context of family health strategy, Paraíba, Brazil. Rev Fac Nac Salud Publica 2019; 37(3):98-109.

17. Machado PMO. Atenção Nutricional no âmbito da Atenção Primária à Saúde: análise de implantação em municípios brasileiros [tese]. Florianópolis: Universidade Federal de Santa Catarina; 2018.

18. Palombo CNT, Fujimori E, Toriyama ATM, Duarte LS. Capacitação em aconselhamento nutricional: avaliação de conhecimento e aplicabilidade na atenção à saúde da criança. Rev Bras Saude Matern Infant 2018; 18(1):75-82.

19. Palombo CNT, Fujimori E, Toriyama ATM, Duarte LS, Borges ALV. Dificuldades no aconselhamento nutricional e acompanhamento do crescimento infantil: perspectivas de profissionais. Rev Bras Enferm 2017; 70(5):1000-1008.

20. Nsiah-Asamoah C. Gaps in knowledge levels of health workers on recommended child feeding practices and growth monitoring and promotion actions. Ped Health Res 2018; 3(2):8.

21. Billah SM, Saha KK, Khan ANS, Chowdhury AH, Garnett SP, Arifeen SE, Menon P. Quality of nutrition services in primary health care facilities: Implications for integrating nutrition into the health system in Bangladesh. PLoS One 2017; 12(5):e0178121.
22. Jaime PC, Santos LMP. Transição nutricional e a organização do cuidado em alimentação e nutrição na atenção básica em saúde. Divulg Saude Debate 2014; 51:72-85.

23. Jaime PC, Delmué DCC, Campello T, Silva DO, Santos LMP. Um olhar sobre a agenda de alimentação e nutrição nos trinta anos do Sistema Único de Saúde. Cien Saude Colet 2018; 23(6):1829-1836.

24. Macinko J, Mendonça CS. Estratégia Saúde da Família, um forte modelo de Atenção Primária à Saúde que traz resultados. Saude Debate 2018; 42(n. esp.):18-37.

25. Pedraza DF. Growth surveillance in the context of the Primary Public Healthcare Service Network in Brazil: literature review. Rev Bras Saude Matern Infant 2016; 16(1):7-19.

26. Rolim MD, Lima SML, Barros DC, Andrade CLT. Avaliação do SISVAN na gestão de ações de alimentação e nutrição em Minas Gerais, Brasil. Cien Saude Colet 2015; 20(8):2359-2369.

27. Nascimento FA, Silva AS, Jaime PC. Cobertura da avaliação do estado nutricional no Sistema de Vigilância Alimentar e Nutricional brasileiro: 2008 a 2013. Cad Saude Publica 2017; 33:e00161516.

28. Nascimento FA, Silva AS, Jaime PC. Cobertura da avaliação do consumo alimentar no Sistema de Vigilância Alimentar e Nutricional Brasileiro: 2008 a 2013. Rev Bras Epidemiol 2019; 22:E190028.

29. Venâncio SI, Martins MCN, Sanches MTC, Almeida H, Rios GS, Frias PG. Análise de implantação da Rede Amamenta Brasil: desafios e perspectivas da promoção do aleitamento materno na atenção básica. Cad Saude Publica 2013; 29(11):2261-2274.

30. Tavares JS, Vieira DS, Dias TKC, Tacla MTGM, Collet N, Reichert APS. Logframe Model as analytical tool for the Brazilian Breastfeeding and Feeding Strategy. Rev Nutr 2018; 31(2):251-262.

31. Ramos AE, Ramos CV, Santos MM, Alemida CAPL, Carvalho e Martins MC. Knowledge of healthcare professionals about breastfeeding and supplementary feeding. Rev Bras Enferm 2018; 71(6):2953-2960.

32. Calvo MCM, Lacerda JT, Colussi CF, Schneider IJC, Rocha TAH. Estratificação de municípios brasileiros para avaliação de desempenho em saúde. Epidemiol Serv Saude 2016; 25(4):767-776.

33. Cardoso AVL, Chain APN, Mendes RIP, Ferreira EF, Vargas AMD, Martins AME, Ferreira RC. Avaliação da gestão da Estratégia Saúde da Família por meio do instrumento Avaliação para Melhoria da Qualidade em municípios de Minas Gerais, Brasil. Cien Saude Colet 2015; 20(4):1267-1284.

34. Fittipaldi ALM, Barros DC, Romano VF. Apoio Matricial nas ações de Alimentação e Nutrição: visão dos profissionais da Estratégia Saúde da Família. Physis 2017; 27(3):793-811.

Artigo apresentado em 23/07/2020

Aprovado em 24/02/2021

Versão final apresentada em 26/02/2021

Editores-chefes: Romeu Gomes, Antônio Augusto Moura da Silva 